University of Wollongong

Research Online

Faculty of Engineering and Information

Faculty of Engineering and Information

Sciences - Papers: Part A

Sciences

$1-1-2015$

\title{
Nonlinear optical conductivity of bilayer graphene with Rashba spin-orbit interaction in the terahertz regime
}

Zheng Liu

Shanghai Institute of Microsystem and Information Technology

Matthew Sanderson

University of Wollongong, ms919@uowmail.edu.au

Chao Zhang

University of Wollongong, czhang@uow.edu.au

J C. Cao

Shanghai Institute of Microsystem and Information Technology

Follow this and additional works at: https://ro.uow.edu.au/eispapers

Part of the Engineering Commons, and the Science and Technology Studies Commons

Research Online is the open access institutional repository for the University of Wollongong. For further information contact the UOW Library: research-pubs@uow.edu.au 


\title{
Nonlinear optical conductivity of bilayer graphene with Rashba spin-orbit interaction in the terahertz regime
}

\author{
Abstract \\ The effect of Rashba spin-orbit coupling on the nonlinear optical conductivity in a bilayer graphene is \\ investigated. We demonstrate the very different role played by the Rashba term and interlayer hopping; in \\ some cases, the two roles can be quite opposite. It is found that the Rashba term can either enhance or \\ suppress the nonlinear effect in a bilayer graphene, depending on the strength of the interlayer hopping. \\ For a weak interlayer hopping, the Rashba term can significantly enhance the nonlinear effect. An \\ analytical result was derived, showing the interplay of the Rashba effect and the interlayer hopping effect.

\section{Disciplines} \\ Engineering | Science and Technology Studies

\section{Publication Details} \\ Liu, Z., Sanderson, M., Zhang, C. \& Cao, J. C. (2015). Nonlinear optical conductivity of bilayer graphene \\ with Rashba spin-orbit interaction in the terahertz regime. Journal of Applied Physics, 118 (4), \\ 043106-1-043106-5.
}




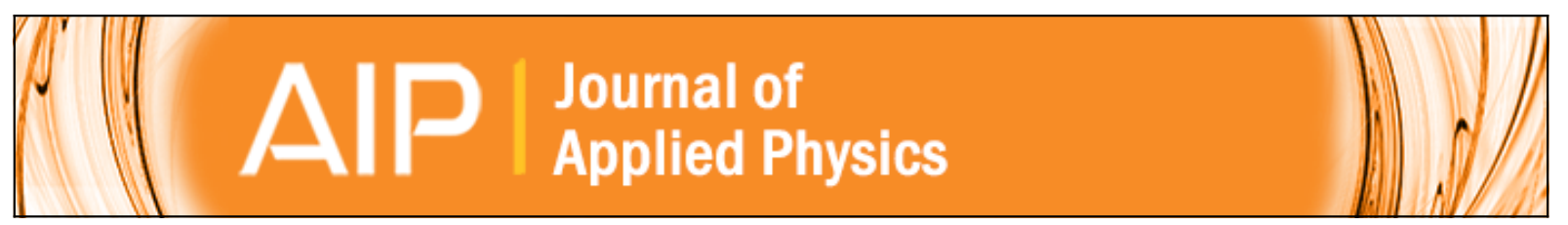

Nonlinear optical conductivity of bilayer graphene with Rashba spin-orbit interaction in the terahertz regime

Zheng Liu, Matthew Sanderson, Chao Zhang, and J. C. Cao

Citation: Journal of Applied Physics 118, 043106 (2015); doi: 10.1063/1.4927512

View online: http://dx.doi.org/10.1063/1.4927512

View Table of Contents: http://scitation.aip.org/content/aip/journal/jap/118/4?ver=pdfcov

Published by the AIP Publishing

\section{Articles you may be interested in}

Spin-orbit force in graphene with Rashba spin-orbit coupling

J. Appl. Phys. 111, 07B330 (2012); 10.1063/1.3678488

Spin-polarized transport in zigzag graphene nanoribbons with Rashba spin-orbit interaction

J. Appl. Phys. 110, 103702 (2011); 10.1063/1.3660704

Graphene p-n junctions with nonuniform Rashba spin-orbit coupling

Appl. Phys. Lett. 99, 162107 (2011); 10.1063/1.3641873

Nonlinear optical spectrum of bilayer graphene in the terahertz regime

Appl. Phys. Lett. 97, 243110 (2010); 10.1063/1.3527934

Nonlinear optical conductance in a graphene pn junction in the terahertz regime

Appl. Phys. Lett. 97, 011907 (2010); 10.1063/1.3462972

\section{SHIMADZU Powerful, Multi-functional UV-Vis-NIR and Excellence in Science FTIR Spectrophotometers}

Providing the utmost in sensitivity, accuracy and resolution for a wide array of applications in materials characterization and nanotechnology research

- Photovoltaics - Ceramics

- Polymers - FPDs

- Thin films

- Coatings

- Paints/inks

- Semiconductors

Click here to learn more
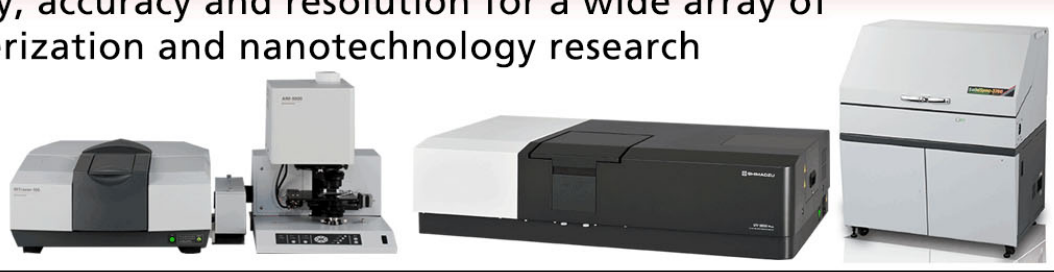


\title{
Nonlinear optical conductivity of bilayer graphene with Rashba spin-orbit interaction in the terahertz regime
}

\author{
Zheng Liu, ${ }^{1}$ Matthew Sanderson, ${ }^{2}$ Chao Zhang, ${ }^{2, a)}$ and J. C. Cao ${ }^{1, b)}$ \\ ${ }^{1}$ Key Laboratory of Terahertz Solid-State Technology, Shanghai Institute of Microsystem and Information \\ Technology, Chinese Academy of Sciences, 865 Changning Road, Shanghai 200050, China \\ ${ }^{2}$ School of Physics, University of Wollongong, New South Wales 2522, Australia
}

(Received 7 May 2015; accepted 16 July 2015; published online 28 July 2015)

\begin{abstract}
The effect of Rashba spin-orbit coupling on the nonlinear optical conductivity in a bilayer graphene is investigated. We demonstrate the very different role played by the Rashba term and interlayer hopping; in some cases, the two roles can be quite opposite. It is found that the Rashba term can either enhance or suppress the nonlinear effect in a bilayer graphene, depending on the strength of the interlayer hopping. For a weak interlayer hopping, the Rashba term can significantly enhance the nonlinear effect. An analytical result was derived, showing the interplay of the Rashba effect and the interlayer hopping effect. @ 2015 AIP Publishing LLC. [http://dx.doi.org/10.1063/1.4927512]
\end{abstract}

\section{INTRODUCTION}

Recently, single-layer graphene (SLG) has attracted considerable attention because of their new physical features, such as the half-integer quantum hall effect, ${ }^{1}$ finite conductivity at zero charge carrier concentration, ${ }^{2}$ perfect quantum tunneling effect, ${ }^{3}$ and ultrahigh carrier mobility. ${ }^{4}$ The band structure of SLG is described at low energies by a twodimensional massless Dirac equation with linear dispersion. This property gives rise to a half integer quantum Hall effect ${ }^{1}$ and dominates the low-energy physics. Shortly after the discovery of graphene, it was observed that bilayer graphene (BLG) also exhibits remarkable phenomena, such as the enhanced optical conductivity, ${ }^{5}$ the terahertz conductivity of twisted bilayer graphene, ${ }^{6}$ and an new type of one-dimensional chiral states. ${ }^{7}$ In BLG, the low-energy excitations are no longer Dirac fermions, like in graphene, but massive chiral fermions due to the parabolicity of the energy bands. In addition, bilayer graphene turns out to be a semiconductor with a gap that can be tuned via a chemical doping or a transverse electric field (gate), which leads to new ways for the confinement of electrons. The BLG is composed of two coupled hexagonal lattices, including inequivalent sites $\mathrm{A}, \mathrm{B}$ and $\tilde{\mathrm{A}}, \tilde{\mathrm{B}}$ in the bottom and top layers, respectively. These are arranged according to Bernal stacking, ${ }^{8}$ so that the A (denoted by $\tilde{\mathrm{A}}$ ) atoms of layer 2 sit directly on top of $\mathrm{B}$ atoms in layer 1, and $\mathrm{A}$ and $\tilde{\mathrm{B}}$ atoms are in the center of the hexagons of the opposing layer and therefore an unit cell of bilayer graphene contains four nonequivalent carbon atoms. Compared to the SLG, the electrons in BLG can hop from A site to B site between two layers with a hopping energy $t_{\perp}$. The electronic properties and the band-structure of the graphene have been intensively studied by the tight-binding method $^{9-11}$ and numerical calculation based on the first principle method, ${ }^{12-14}$ or the coherent-potential approximation. ${ }^{15}$ When the spin-orbit interaction (SOI) is introduced into the system because of the structure inversion asymmetry due to

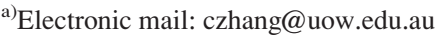

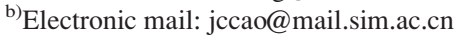

the presence of impurities or external electric gate voltages, the band-structure is strongly modified and the topology of the energy bands changes. Under the Rashba spin-orbit interaction (RSOI), which is an external SOI, the low energy bands undergo trigonal warping deformation at the $K$ and $K^{\prime}$ points of the graphene's Brillouin zone (BZ) in the reciprocal lattice. One Dirac point is now split into four gapless points. ${ }^{16-20}$ One of them remains at the $K\left(K^{\prime}\right)$ point and other three link up an equilateral triangle around the $K\left(K^{\prime}\right)$ point. The warping changes the topology of the low energy bands at the $K\left(K^{\prime}\right)$ point of the graphene's BZ. ${ }^{19,21}$

On other hand, it has been proven that graphene can manifest strong nonlinear effect and has the strong optical response in the terahertz regime. ${ }^{22-24}$ For the SLG, the linear conductivity with RSOI at low frequency has been studied recently. ${ }^{25}$ The third order nonlinear conductivity with RSOI has also been studied recently and it is reported that the third order nonlinear conductivity for SLG can be enhanced depending on the coupling Rashba strength, ${ }^{26}$ which originates from the change of the topology of the energy bands. The BLG system which can be considered as two coupled SLGs (Ref. 8) has the interlayer Rashba interaction depending on the orientation of this electric field as well as the interlayer hopping energy $t_{\perp}$. In comparison to the case of SLG, more hopping terms are involved in the BLG, which make the band structure more complicated than in the SLG. It is discussed in detail in Refs. 17 and 27 with many effects taken into account. In this paper, we investigate the effect on the third order nonlinear conductivity of the BLG by the RSOI. To capture the main features resulting from the interlayer hopping and RSOI, we ignore other non-vertical interlayer hopping terms which are one order of magnitude smaller than $t_{\perp}$, also ignore the scattering by phonons ${ }^{28}$ and impurities. ${ }^{29}$

\section{THEORY AND MODEL}

\section{A. The electronic properties}

The creation operator of the basis for the BLG is defined as 


$$
\begin{aligned}
\hat{\psi}^{\dagger}(k)= & \left\{\hat{C}_{A, \uparrow, 1}^{\dagger}(k), \hat{C}_{A, \downarrow, 1}^{\dagger}(k), \hat{C}_{B, \uparrow, 1}^{\dagger}(k), \hat{C}_{B, \downarrow, 1}^{\dagger}(k)\right. \\
& \left.\times \hat{C}_{A, \uparrow, 2}^{\dagger}(k), \hat{C}_{A, \downarrow, 2}^{\dagger}(k), \hat{C}_{B, \uparrow, 2}^{\dagger}(k), \hat{C}_{B, \downarrow, 2}^{\dagger}(k)\right\} .
\end{aligned}
$$

The Hamiltonian of the BLG system in the tight-bind approximation can be written as

$$
\hat{H}=\sum_{k} \hat{\psi}^{\dagger}(k) M_{8 \times 8} \hat{\psi}(k),
$$

where

$$
M_{8 \times 8}=\left(\begin{array}{cc}
V \mathscr{I}_{4 \times 4}+M_{4 \times 4}^{\text {intra }} & M_{\perp} \\
M_{\perp}^{\dagger} & -V \mathscr{I}_{4 \times 4}+M_{4 \times 4}^{\text {intra }}
\end{array}\right),
$$

with $M_{4 \times 4}^{\text {intra }}=M_{4 \times 4}^{H_{0}+H_{R S O I}}+M_{4 \times 4}^{H_{I S O}}$. The model includes the intrinsic spin-orbit (ISO) interaction, RSOI, and the gate voltage $\mathrm{V}$. The gate voltage can be tuned externally such that the lower layer has an electric potential V, while the upper layer has $-\mathrm{V}$. The relative matrices are follows:

$$
\begin{gathered}
M_{\perp}=\left(\begin{array}{cccc}
0 & 0 & t_{\perp} & 0 \\
0 & 0 & 0 & t_{\perp} \\
0 & 0 & 0 & 0 \\
0 & 0 & 0 & 0
\end{array}\right), \\
M_{4 \times 4}^{H_{0}+H_{R S O I}}=\left(\begin{array}{cccc}
0 & 0 & \varphi_{0} & i \varphi_{+} t_{R} \\
0 & 0 & i \varphi_{-} t_{R} & \varphi_{0} \\
\varphi_{0}^{*} & -i \varphi_{-}^{*} t_{R} & 0 & 0 \\
-i \varphi_{+}^{*} t_{R} & \varphi_{0}^{*} & 0 & 0
\end{array}\right), \\
M_{4 \times 4}^{H_{I S O}}=t_{I S O}\left(\begin{array}{cccc}
\eta_{k} & 0 & 0 & 0 \\
0 & -\eta_{k} & 0 & 0 \\
0 & 0 & -\eta_{k} & 0 \\
0 & 0 & 0 & \eta_{k}
\end{array}\right)
\end{gathered}
$$

where $\varphi_{0}=e^{\frac{i a k_{y}}{\sqrt{3}}}\left[1+2 e^{-\frac{1}{2} i \sqrt{3} a k_{y}} \cos \left(\frac{a k_{x}}{2}\right)\right], \varphi_{+}=e^{\frac{i a k_{y}}{\sqrt{3}}}\left[1-2 e^{-\frac{1}{2} i \sqrt{3}}\right.$ $\left.a k_{y} \sin \left(\frac{a k_{x}}{2}+\frac{\pi}{6}\right)\right], \varphi_{-}=e^{\frac{i a k_{y}}{\sqrt{3}}}\left[1+2 e^{-\frac{1}{2} i \sqrt{3} a k_{y}} \sin \left(\frac{a k_{x}}{2}-\frac{\pi}{6}\right)\right] \cdot t_{R}, t_{I S O}$ are the intralayer coupling energies for the RSOI and the ISO in the unit of the hopping energy $t_{h} \simeq 2.7 \mathrm{eV}$. $t_{\perp}$ is the interlayer hopping energy between $\mathrm{A}$ and $\tilde{\mathrm{A}}$ atom and $a$ is the lattice constant.

According to Ref. 17, it is found that the intralayer Rashba coupling in an unbiased bilayer not only splits the $\mathrm{K}$ $\left(\mathrm{K}^{\prime}\right)$ point into four, in a different way than it does for monolayer graphene, ${ }^{18}$ but also creates a Dirac cone out of a parabolic band. In addition, a Mexican-hat band arises at the $\mathrm{K}$ $\left(\mathrm{K}^{\prime}\right)$ point when the pure SOI interactions exist or the layers are solely biased. Because it is the gapless cone that the low energy Dirac equation characterizes, it is assumed that $t_{I S O}=V=0$ such that the gap vanishes in the following analysis. In Fig. 1, the energy spectrum of bilayer graphene along the line $k_{y}=\frac{2 \pi}{\sqrt{3} a}$ is given with the parameters $t_{\perp}=0.2$, $t_{R}=0.2$, and the geometry of the band structure around the point $K=\left(\frac{2 \pi}{3}, \frac{2 \pi}{\sqrt{3}}\right) \frac{1}{a}$ is shown in the inset. Besides the four Dirac cones created due to the RSOI same as the case of

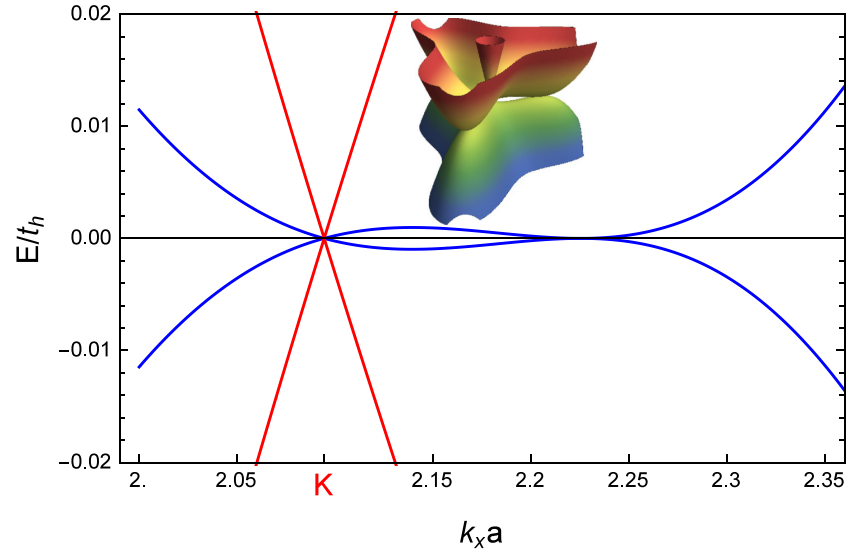

FIG. 1. Energy spectrum of bilayer graphene along the line $k_{y}=\frac{2 \pi}{\sqrt{3} a}$ with the parameters $t_{R}=0.2, t_{\perp}=0.2, t_{h}=1$. Inset: The band structure around the point $\mathrm{K}$ with the same parameters.

SLG (blue line), an external Dirac cone occurs with the larger slope (red line), which is different from the case of SLG. Fig. 2 gives the energy bands of the BLG, which include the new Dirac cones for the different interlayer hopping energies $t_{\perp}$ with a fixed intralayer RSOI energy $t_{R}=0.2$. For the case of SLG with the nonzero RSOI, which breaks the $\mathrm{SU}(2)$ symmetry of the system, the twofold spin degeneracy is lifted except some special high symmetry point like $K\left(K^{\prime}\right)$ (essential degeneracy) and the point of accidental degeneracy dependent on the parameter $t_{R}$. When the interlayer hopping energy $t_{\perp}$ occurs (imaging the two isolated SLG close to each other from infinity), the hopping term lifts the accidental degeneracy and creates the new band, including an additional Dirac cone (ADC) (the branches with larger slope) as shown in Fig. 2. Like the case of the SLG with RSOI where the new Dirac cones result from the change of the topology of the band, the new Dirac cones in the case of BLG are also created due to the interlayer hopping energy $t_{\perp}$, which cause the change of the topology of the bands of the double SLGs system (not BLG) with RSOI. When the interlayer hopping energy $t_{\perp}$ is fixed, changing the intralayer RSOI energy, $t_{R}$ also has an effect on the band but it is different from the case of changing $t_{\perp}$.

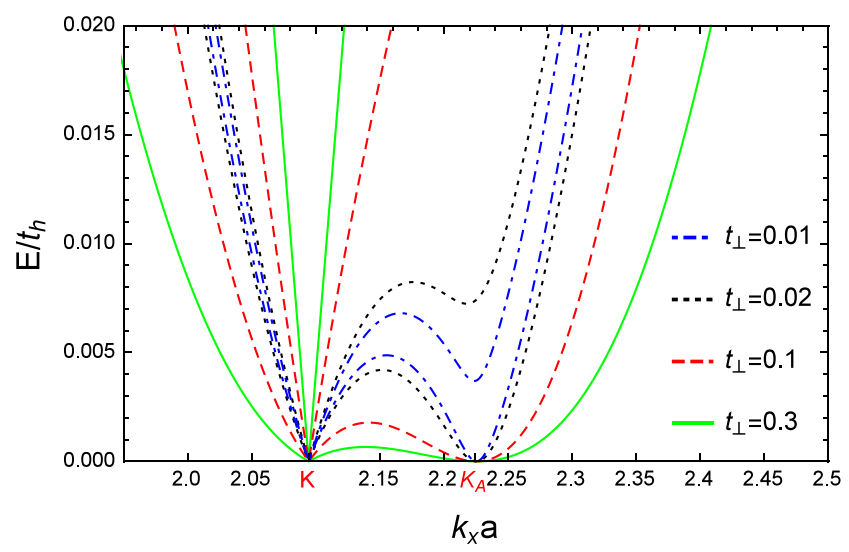

FIG. 2. The bands including the Dirac cones along the line $k_{y}=\frac{2 \pi}{\sqrt{3} a}$ for the different interlayer hopping energies $t_{\perp}=0.3,0.1,0.02,0.01$. The fixed RSOI strength $t_{R}=0.2$. 


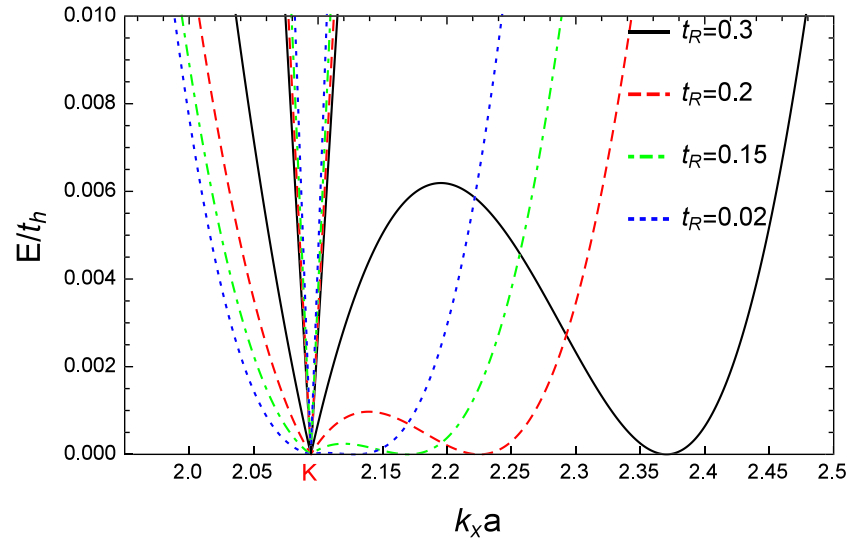

FIG. 3. The bands including the Dirac cones along the line $k_{y}=\frac{2 \pi}{\sqrt{3} a}$ with the interlayer hopping $t_{\perp}=0.2$ and the different RSOI strengths $t_{R}=0.3,0.2$, $0.15,0.02$.

The bands of the BLG for different values of $t_{R}$ with the fixed $t_{\perp}=0.2$ are shown in Fig. 3. It follows that with the fixed $t_{\perp}$ that is responsible for the existence of ADC, the point of the accidental degeneracy shifts to $\mathrm{K}$ point, the height of cone is reduced and vanishes finally when the intralayer RSOI descends. However, the ADC is not dramatically affected and it is implicated that there seems a contrary effect on the height of the Dirac cones between $t_{R}$ and $t_{\perp}$.

\section{B. The third order nonlinear conductivity from a Dirac cone}

For the electronic system where the geometry of the energy band can be described by the Dirac cone, when it interacts with the external fields $\vec{A}=\vec{A}_{0} e^{-i \omega t}$, the effective interacting system is described by the effective Hamiltonian

$$
\hat{H}(p)=v_{F}\left(\begin{array}{cc}
0 & p_{-}+e A_{-} \\
p_{+}+\mathrm{eA}+ & 0
\end{array}\right),
$$

where $A_{ \pm}=A_{x} \pm i A_{y}, p_{ \pm}=p_{x} \pm i p_{y}$. For simplicity, it is assumed that $A_{y}=E_{y}=0$ and then $A_{-}=A_{+}=A_{x}=\frac{E_{x}}{i \omega}=\frac{E}{i \omega}$ $\exp ^{-i \omega t}$ with $\left|E_{x}\right| \equiv E$. The electron on the Fermi-surface of cone-like shape is modeled by the two-dimensional lower energy Dirac-like quasi-particle with the dispersion relation $E(k)=\hbar v_{F} k$. The basis vectors of the representation space are the Bloch vectors $\left|\varphi_{\nu}(\vec{k})\right\rangle=\frac{1}{\sqrt{N}} \sum_{\vec{l}_{\nu} \in T_{\nu}} e^{i \vec{k} \cdot \vec{l}_{\nu}} \phi_{2 p_{z}}\left(\vec{r}-\vec{l}_{\nu}\right)$ $\equiv\left\langle\vec{r}\left|\hat{C}_{\nu}^{\dagger}(k)\right| 0\right\rangle(\nu=A, B)$ and $\phi_{2 p_{z}}(\vec{r})$ is usually chosen as the $2 p_{z}$ orbit wave function of the carbon atom. According to the Floquet theorem, the time-dependent two-component wave functions are expanded as $\psi(p, t)=\sum_{n=0}^{\infty}\left[\begin{array}{c}\alpha_{n}(p) \\ \beta_{n}(p)\end{array}\right] e^{i n \omega t} e^{-i \varepsilon t / \hbar}$.

After substituting it into the Schrödinger equation, the recursive equations of the components can be obtained ${ }^{22,23}$ and the excited current by the external field $\vec{A}(t)$ is given by

$$
\vec{J}=\frac{e}{4 \pi^{2} \hbar^{2}} \int\left\langle\vec{v}\left(p_{x}, p_{y}\right)\right\rangle N[\varepsilon(p)] d p_{x} d p_{y}
$$

where $\hat{\vec{v}}=\frac{\partial \hat{H}(p)}{\partial \vec{p}},\langle\vec{v}(p)\rangle=\psi^{\dagger} \hat{\vec{v}} \psi$, and $N(\varepsilon)=n_{F}(-\varepsilon)-n_{F}(\varepsilon)$ is the thermal factor. From Eq. (8), the third order nonlinear conductivity is extracted and given by $\sigma_{3}^{D}=\frac{e^{2}}{4 \hbar} \frac{e^{2} E_{0}^{2}}{\hbar^{2} \omega^{4}}$ $\left[N_{3}(\omega) e^{i 3 \omega t}+N_{1}(\omega) e^{i \omega t}\right] v_{F}^{2}$. The detailed derivations can be found in Refs. 22 and 23. For the case of BLG, the base vectors become $\left|\varphi_{\nu, 1}(\vec{k})\right\rangle,\left|\varphi_{\nu, 2}(\vec{k})\right\rangle(\nu=A, B)$ and the representation space at a fixed $\vec{k}$ is four-dimensional. It is the direct sum of the subspace of two isolated SLGs with the freedom of spin ignored. For the double SLGs system coupled only by $t_{\perp}$ interaction, there does not exist degeneracy at $K$ as shown in Fig. 4(a). However, when the RSOI is taken into account in the double SLGs system, the degeneracy occurs at $K$ point (shown in Fig. 4(b)) and a new Dirac cone is created. It may seem a little counter-intuitive in comparison with the case of SLG with RSOI.

For a Dirac cones with a dispersion relation $E(k)=\hbar v_{F} k$, the standard slope is $\frac{d \tilde{E}}{d \tilde{k}}=\frac{\hbar v_{F}}{a t_{h}}=\frac{\sqrt{3}}{2} \equiv \alpha_{s}$ with the dimensionless energy $\tilde{E}=E / t_{h}$ and $\tilde{k}=k a$ used. For the Dirac cone of the BLG, they can be measured relative to the standard Dirac cone by the ratio of its slope $\alpha\left(t_{h}, t_{R}\right)$ to $\alpha_{s}$. Define the relative slope of the Dirac cone $\beta\left(t_{h}, t_{R}\right) \equiv \frac{\alpha\left(t_{h}, t_{R}\right)}{\alpha_{s}}=\frac{2 \sqrt{3} \alpha}{3}$ and therefore, the effective Fermi velocity of the Dirac cone is $v_{F}^{e f f}\left(t_{h}, t_{R}\right)=\beta v_{F}$. If we ignore the effect of the shape-change of cone and limit the energy of the incoming photon $\hbar \omega$ less than the height of the cones, which is topologically determined to be valid for our linear model, we obtain the third order nonlinear conductivity of the BLG with RSOI

$$
\sigma_{3, R S O I}^{B L G}=6\left(\beta_{A D C}^{2}+\beta_{0}^{2}+3 \beta_{1}^{2}\right) \sigma_{3}^{D},
$$

where $\beta_{A D C}\left(t_{h}, t_{R}\right)$ and $\beta_{0}\left(t_{h}, t_{R}\right)$ are the relative slopes of the cones at $K$, and $\beta_{1}\left(t_{h}, t_{R}\right)$ is the relative slope of the three
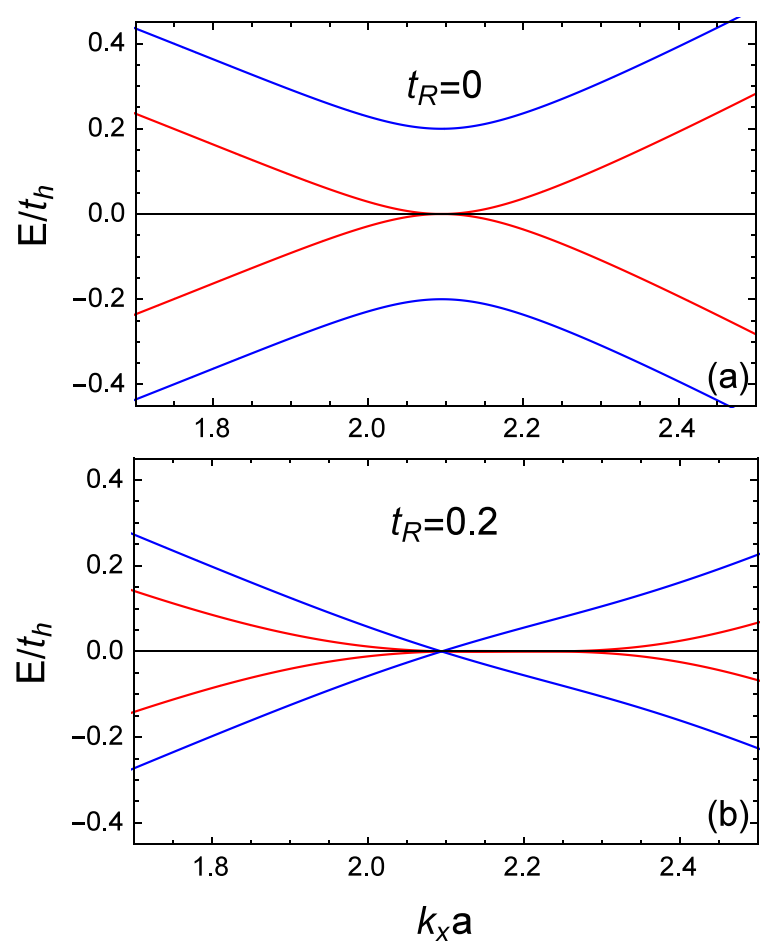

FIG. 4. The bands around the point $K$ along the line $k_{y}=\frac{2 \pi}{\sqrt{3} a}$ the interlayer hopping $t_{\perp}=0.2$ (a) without RSOI: $t_{R}=0$ (b) with RSOI: $t_{R}=0.2$. 


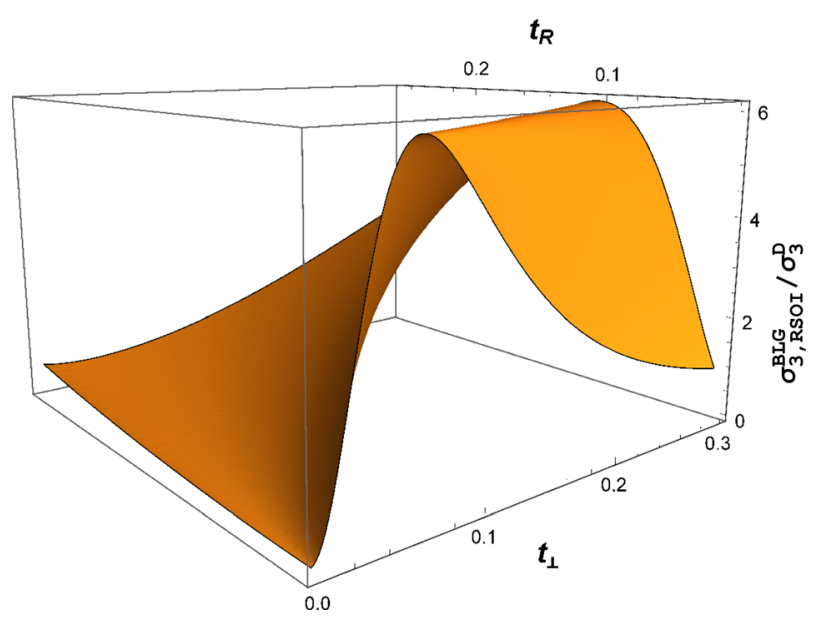

FIG. 5. Third order nonlinear conductivity relative to $\sigma_{3}^{D}$ for the parameters pair $\left(t_{R}, t_{\perp}\right)$.

satellite cones created by the RSOI, which is similar as the case of SLG.

\section{RESULTS AND DISCUSSIONS}

In terms of Eq. (9) and the numerically calculated slopes, we obtain the nonlinear conductivity in the range of $t_{\perp}, t_{R}$ that are about $t_{R} / t_{h} \sim 0.25$ and $t_{\perp} / t_{h} \sim 0.3$, which is easily achievable, and the results are shown in Fig. 5. When the coupling energies $t_{\perp}, t_{R}$ vary, the height of the cone $E_{H C}\left(t_{\perp}\right.$, $t_{R}$ ) (the dome with blue line in Fig. 1) also changes. The height is the upper limit of the frequency of the permitted incoming photon. The linear model holds only for the photon the energy of which is lower than the height of the cone. In Fig. 6, the contour of $E_{H C}\left(t_{\perp}, t_{R}\right)$ is given in the terahertz range for the practical purpose. In Fig. 7, the nonlinear conductivity relative to $\sigma_{3}^{D}$, which is the nonlinear conductivity of the SLG without RSOI, is shown for the different $t_{\perp}, t_{R}$ by numerically calculating the slopes of the cones. It is found from the figure that when $t_{\perp}$ or $t_{R}$ is fixed, there exist a maximum of the nonlinear conductivity that is close to 6 times of $\sigma_{3}^{D}$. It is very different from the case of SLG where the nonlinear conductivity with the RSOI ascends monotonously

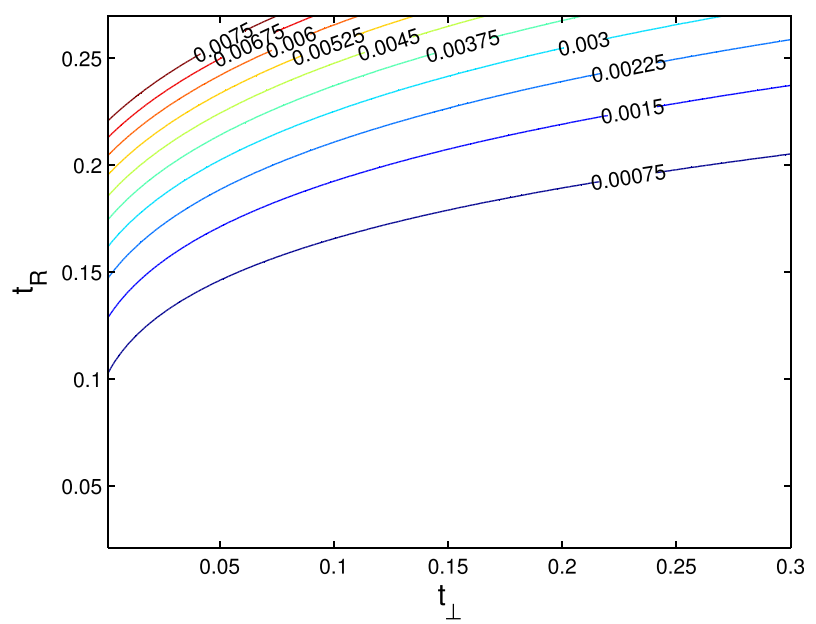

FIG. 6. The contour of the energy height of the cone corresponding to the parameters pair $\left(t_{R}, t_{\perp}\right)$.
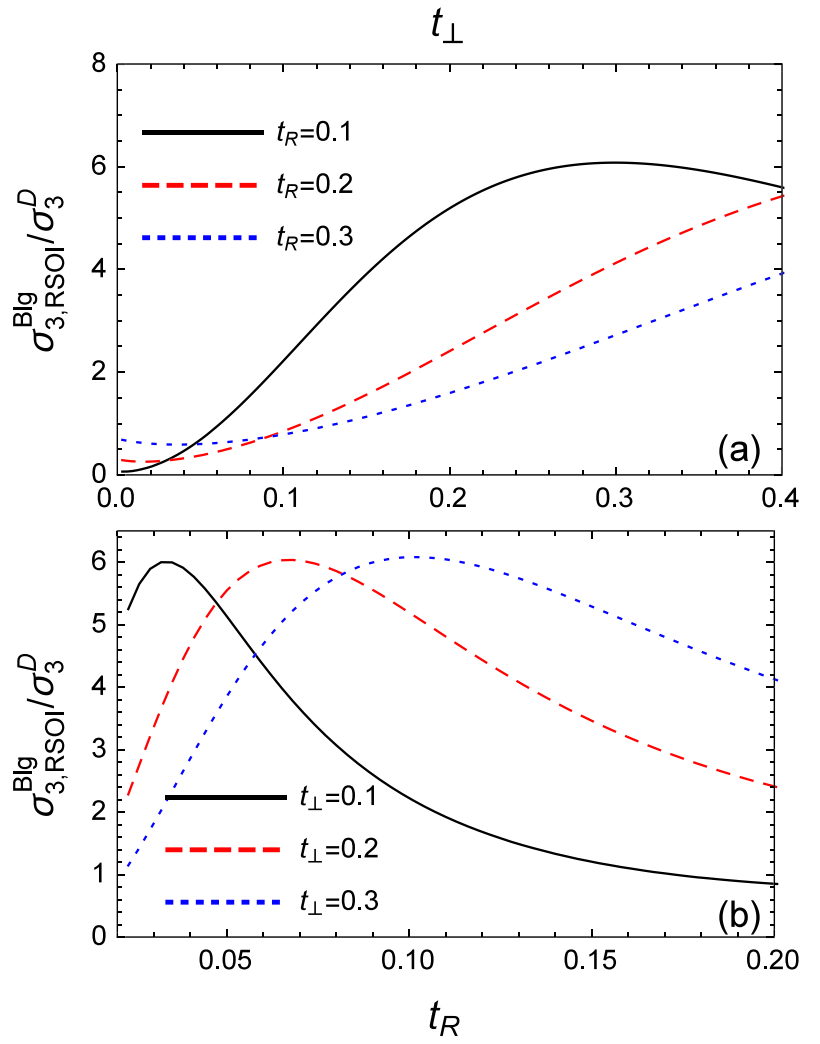

FIG. 7. The third order nonlinear conductivity relative to $\sigma_{3}^{D}$ for different $t_{\perp}$, $t_{R}$. (a) Curve of $\sigma_{3, R S O I}^{B L G} \sim t_{\perp}$ for the different $t_{R}$. (b) Curve of $\sigma_{3, R S O I}^{B L G} \sim t_{R}$ for the different $t_{\perp}$.

with the increase in $t_{R}$. It is noted from Fig. 7(b) that when the interlayer hopping term $t_{\perp}$ increases, the peak of curve $\sigma_{3, B L G}^{R S O I} \sim t_{R}$ shifts to the right, which suggests that for a small $t_{R}$, the height of the Dirac cone makes a decisive effect on the nonlinear conductivity $\sigma_{3, B L G}^{R S O I}$ and the height is reduced as $t_{\perp}$ increases (Fig. 2). Physically, increasing $t_{\perp}$ means that the probability of interlayer hopping increases and more electrons transverse vertically. This would cause the intralayer current to be distracted. On the other hand, the large RSOI tends to stick the electron in plane, which is opposite to the role played by $t_{\perp}$. However, after an electron hops into one of the single-layers vertically, it is possible that it subsequently integrates into the intralayer current. If the process of the nonlinear resonance and the vertical hopping properly matches, it will lead to an enhancement of the intralayer current of the layer that it plunges into and contribute the peak of the nonlinear conductivity shown in Fig. 7. This property that the nonlinear conductivity is determined by the vertically hopping process and RSOI together facilitate the control of the optical device that is based on the graphene, for instance, the single-photon nonlinear optical cavity with graphene plasmons ${ }^{30}$ where the nonlinearity of the cavity can be tuned by the above effect.

In conclusions, the nonlinear conductivity of the BLG with RSOI is explored with the vertically hopping taken into account. The results show that the nonlinear conductivity of the BLG can be enhanced in comparison with the case without RSOI when $t_{R}$ is a small parameter. There exists a peak $\sigma_{3, B L G}^{R S O I}\left(t_{R}^{\max }\right)$ for the nonlinear conductivity to the parameter 
$t_{R}$. Once $t_{R}>t_{R}^{\max }$, the nonlinear conductivity is reduced. Moreover, the process of the vertically hopping plays an important role in the nonlinear response process and can interact with the process of RSOI. Compared with the case of SLG where there is no vertically hopping, the interaction between RSOI and the vertically hopping increases the nonlinear conductivity of BLG relative to the one in SLG with RSOI if the "phase" of the two physical processes matches each other. In the case of SLG, it is known that the RSOI can cause the change of the topology of the band and create new Dirac cones that bring additional contribution to the nonlinear conductivity, but the effective Fermi velocity of the ADC in SLG is less than the Fermi velocity $v_{F}$ of the original Dirac cone. In the BLG, the vertically hopping can facilitate the increase in the effective Fermi velocity of the ADC. There are other factors, such as the bias voltage $V$, interlayer SOI interaction, and other hopping parameters, which may have an effect on the shape of the Dirac cone in different degrees.

\section{ACKNOWLEDGMENTS}

This work was supported by the 973 Program of China (Grant No. 2014CB339803), the 863 Program of China (Project No. 2011AA010205), the National Natural Science Foundation of China (Grant Nos. 61131006, 61321492, and 11174309) the Major National Development Project of Scientific Instrument and Equipment (Grant No. 2011YQ150021), the National Science and Technology Major Project (Grant No. 2011ZX02707), the major project (Project No. YYYJ-1123-1), the International Collaboration and Innovation Program on High Mobility Materials Engineering of the Chinese Academy of Sciences, and the Australian Research Council Grant through a Discovery Grant (DP140101501).

${ }^{1}$ Y. Zhang, Y. W. Tan, H. L. Stormer, and P. Kim, Nature (London) 438, 201 (2005).
${ }^{2}$ K. S. Novoselov, A. K. Geim, S. V. Morozov, D. Jiang, M. I. Katsnelson, I. V. Grigorieva, S. V. Dubonos, and A. A. Firsov, Nature (London) 438, 197 (2005).

${ }^{3}$ M. I. Katsnelson, K. S. Novoselov, and A. K. Geim, Nat. Phys. 2, 620 (2006).

${ }^{4}$ A. K. Geim and K. S. Novoselov, Nature Mater. 6, 183 (2007).

${ }^{5}$ A. R. Wright, J. C. Cao, and C. Zhang, Phys. Rev. Lett. 103, 207401 (2009).

${ }^{6}$ X. Q. Zou et al., Phys. Rev. Lett. 110, 067401 (2013).

${ }^{7}$ I. Martin, Ya. M. Blanter, and A. F. Morpurgo, Phys. Rev. Lett. 100, 036804 (2008).

${ }^{8}$ E. McCann and V. I. Fal'ko, Phys. Rev. Lett. 96, 086805 (2006).

${ }^{9}$ P. R. Wallace, Phys. Rev. 71, 622 (1947).

${ }^{10}$ S. Reich, J. Maultzsch, and C. Thomsen, Phys. Rev. B 66, 035412 (2002).

${ }^{11}$ A. Grneis, C. Attaccalite, L. Wirtz, H. Shiozawa, R. Saito, T. Pichler, and A. Rubio, Phys. Rev. B 78, 205425 (2008).

${ }^{12}$ R. C. Tatar and S. Rabii, Phys. Rev. B 25, 4126 (1982).

${ }^{13}$ J.-C. Charlier, X. Gonze, and J.-P. Michenaud, Phys. Rev. B 43, 4579 (1991).

${ }^{14}$ H. Min, B. Sahu, S. K. Banerjee, and A. H. MacDonald, Phys. Rev. B 75, 155115 (2007).

${ }^{15}$ J. Nilsson, A. H. Castro Neto, F. Guinea, and N. M. R. Peres, Phys. Rev. B 78, 045405 (2008).

${ }^{16}$ P. Rakyta, A. Kormányos, and J. Cserti, Phys. Rev. B 82, 113405 (2010).

${ }^{17}$ R. van Gelderen and C. M. Smith, Phys. Rev. B 81, 125435 (2010).

${ }^{18}$ M. Zarea and N. Sandler, Phys. Rev. B 79, 165442 (2009).

${ }^{19}$ M. Gmitra, S. Konschuh, C. Ertler, C. Ambrosch-Draxl, and J. Fabian, Phys. Rev. B 80, 235431 (2009).

${ }^{20}$ S. Konschuh, M. Gmitra, and J. Fabian, Phys. Rev. B 82, 245412 (2010).

${ }^{21}$ T. Fukui and Y. Hatsugai, Phys. Rev. B 75, 121403(R) (2007).

${ }^{22}$ A. R. Wright, X. G. Xu, J. C. Cao, and C. Zhang, Appl. Phys. Lett. 95, 072101 (2009).

${ }^{23}$ X. G. Xu, S. Sultan, C. Zhang, and J. C. Cao, Appl. Phys. Lett. 97, 011907 (2010).

${ }^{24}$ C. Zhang, L. Chen, and Z. Ma, Phys. Rev. B 77, 241402 (2008).

${ }^{25}$ W. Wang, C. Zhang, and Z. Ma, J. Phys.: Condens. Matter 24, 035303 (2012).

${ }^{26}$ Z. Liu, M. Sanderson, J. C. Cao, and C. Zhang, Phys. Rev. B 90, 235430 (2014).

${ }^{27}$ S. Konschuh, M. Gmitra, D. Kochan, and J. Fabian, Phys. Rev. B 85, 115423 (2012).

${ }^{28}$ C. Zhang and Y. Takahashi, J. Phys.: Condens. Matter 5, 5009 (1993).

${ }^{29}$ N. Tzoar and C. Zhang, Phys. Rev. B 32, 1146 (1985).

${ }^{30}$ M. Gullans, D. E. Chang, F. H. L. Koppens, F. J. García de Abajo, and M. D. Lukin, Phys. Rev. Lett. 111, 247401 (2013). 